EPJ manuscript No.

(will be inserted by the editor)

\title{
Magnetic Anisotropy of Deposited Transition Metal Clusters
}

\author{
S. Bornemann ${ }^{1}$, J. Minár ${ }^{1}$, J. B. Staunton ${ }^{2}$, J. Honolka ${ }^{3}$, A.Enders ${ }^{3}$, K. Kern ${ }^{3}$ and H. Ebert ${ }^{1}$ \\ 1 Department Chemie und Biochemie, Ludwig-Maximilians-Universität München, 81377 München, Germany \\ 2 Department of Physics, University of Warwick, Coventry CV4 7AL, United Kingdom \\ 3 Max Planck Institut for Solid State Research, 70569 Stuttgart, Germany
}

Received: / Revised version:

\begin{abstract}
We present results of magnetic torque calculations using the fully relativistic spin-polarized Korringa-Kohn-Rostoker approach applied to small Co and Fe clusters deposited on the Pt(111) surface. From the magnetic torque one can derive among others the magnetic anisotropy energy (MAE). It was found that this approach is numerically much more stable and also computationally less demanding than using the magnetic force theorem that allows to calculate the MAE directly. Although structural relaxation effects were not included our results correspond reasonably well to recent experimental data.
\end{abstract}

PACS. 73.22.-f - 75.30.Et $-75.75 .+\mathrm{a}$

\section{Introduction}

In recent years, low-dimensional magnetic nanostructures on surfaces have been the subject of intense experimental and theoretical research activities which are driven by fundamental as well as practical interests. One of the central questions for potential future applications is how the spin-orbit coupling (SOC) induces specific magnetic properties such as orbital magnetic moments and magnetic anisotropy. Another interesting issue is its influence on the exchange coupling. Hereby, it is especially important to understand how these properties evolve from single magnetic adatoms to submonolayer magnetic particles. This very prominent role of SOC for such systems is also reflected in recent reviews [1] as well as theoretical work [2].

A system which has been intensively investigated experimentally $3456 / 7 / 8910$ as well as theoretically 1112 in recent years is $\mathrm{Co} / \mathrm{Pt}(111)$ (used here as a short notation for Co clusters or nanostructures, respectively, deposited on a $\mathrm{Pt}(111)$ substrate) as this is a prototype to study the requirements on new high-density magnetic storage materials. Earlier theoretical works studied only rather small Co clusters or Co chains on Pt(111) 1112 1314 whereas only recently first qualitative results and trends based on a parameterised tight-binding approach were published for deposited structures of up to 37 Co atoms [15]. In our earlier works 111213 on the $\mathrm{Co} / \mathrm{Pt}(111)$ system we already described the evolution of the spin and orbital moments as well as the exchange coupling for small clusters. What has been missing so far, for a complete picture of the magnetic behaviour is the magnetic anisotropy energy (MAE) for such systems. In principle, calculations of the MAE are possible by applying the magnetic force theorem or by determining the total energies as a function of the magnetisation direction. However, it turned out that these procedures are rather delicate when dealing with deposited clusters and one needs to take great care when taking band or total energy differences. Therefore, we implemented a method to calculate the magnetic torque directly from the electronic structure. Calculating the magnetic torque for a sequence of directions of the magnetisation then gives access to the MAE.

In this present work we show first results for magnetic torque calculations of the already well investigated Co adatoms and dimers deposited on $\mathrm{Pt}(111)$ and compare them with their $\mathrm{Fe}$ analogues. These investigations are complemented by calculations for decorated clusters that allow to optimize the MAE. Our theoretical results are then used to simulate magnetisation curves of an ensemble of $\mathrm{Fe}_{\mathrm{n}}(\mathrm{n}=1,2,3)$ clusters on $\mathrm{Pt}(111)$, that are compared 131415 t experimental results.

\section{Computational Details}

The calculations for the investigated cluster systems were ,done within the framework of spin density functional theory using the local spin density approximation (LSDA) with the parameterization given by Vosko, Wilk and $\mathrm{Nu}-$ sair for the exchange and correlation potential 16. The electronic structure is determined in a fully relativistic way on the basis of the Dirac equation for spin-polarised potentials which is solved using the Korringa-Kohn-Rostoker (KKR) multiple scattering formalism 17,18. This procedure consists of two steps. First the $\mathrm{Pt}(111)$ host surface is calculated self-consistently with the tight binding (TB) version of the KKR method using layers of empty sites 
to represent the vacuum region. This step is then followed by treating the deposited clusters as a perturbation to the clean surface with the Green's function for the new system being obtained by solving the corresponding Dyson equation. This scheme is described in more detail in earlier publications [12[13].

In all calculations the cluster atoms were assumed to occupy ideal lattice sites in the first vacuum layer and no effects of structure relaxation were included. Therefore, our results contain a systematic error and are strictly spoken not directly comparable with experimental data. Nevertheless, it could be shown already in earlier work 1113 14 on deposited clusters that this approach is capable of reproducing systematic trends as well as achieving a reasonable quantitative agreement with values found in experiment.

The $\alpha_{\hat{u}}$-component of the torque vector $T_{\alpha_{\hat{u}}}^{(\hat{n})}$ on the magnetic moments oriented along the direction $\hat{n}$ was calculated with an expression based on Lloyd's formula and perturbation theory [19]

$$
\begin{aligned}
& T_{\alpha_{\hat{u}}}^{(\hat{n})}=-\frac{1}{\pi} \int d E f_{F D}(E) \\
& \operatorname{Im} i \sum_{i} \operatorname{tr}\left(\underline{\tau}_{i i}^{(\hat{n})}\left[(\hat{u} \cdot \underline{\hat{J}}) \underline{t}_{i}^{(\hat{n})^{-1}}-\underline{t}_{i}^{(\hat{n})^{-1}}(\hat{u} \cdot \underline{\hat{J}})\right]\right) .
\end{aligned}
$$

Here $f_{F D}$ is the Fermi distribution function, $\hat{u}$ is the direction of the torque vector and $\hat{J}$ is the total angular momentum operator. Finally, the matrices $\underline{t}_{i}^{(\hat{n})}$ and $\underline{\tau}_{i i}^{(\hat{n})}$ are the single site t-matrix and the site diagonal scattering path operator, respectively, where $i$ (used as an index) labels the atomic sites. As the calculations are done assuming the temperature $\mathrm{T}=0 \mathrm{~K}$ the Fermi distribution function is replaced by the theta function $\Theta\left(E_{F}-E\right)$ with $E_{F}$ being the Fermi energy. For all results shown below, the energy integral $\int d E$ occuring in Eq. (11) was calculated on a rectangular complex energy mesh containing 64 points, while using an angular momentum expansion up to $l_{\max }=2$ for all the occuring matrices.

Eq. (1) uses the analytic derivative of the energy with respect to a rotation angle. We found that this approach is numerically much more robust than taking the differences between band or total energies. The disadvantage, however, is that the magnetic anisotropy energy, defined as the difference $E\left(\hat{n}, \hat{n}_{0}\right)$ of the energy for two orientations of the magnetisation, $\hat{n}$ and $\hat{n}_{0}$, respectively, has to be determined by a corresponding path integral:

$$
E\left(\hat{n}, \hat{n}_{0}\right)=\int_{\hat{n}_{0}}^{\hat{n}} T^{(\hat{n})} d \hat{n} .
$$

Developing $E\left(\hat{n}, \hat{n}_{0}\right)$ in spherical harmonics up to second order and taking into account the symmetry of the investigated cluster substrate systems, one finds for example for a cluster having $\mathrm{C}_{2 \mathrm{v}}$-symmetry with respect to its spatial structure, i.e. ignoring the orientation $\hat{n}$ of the magnetisation [20]:

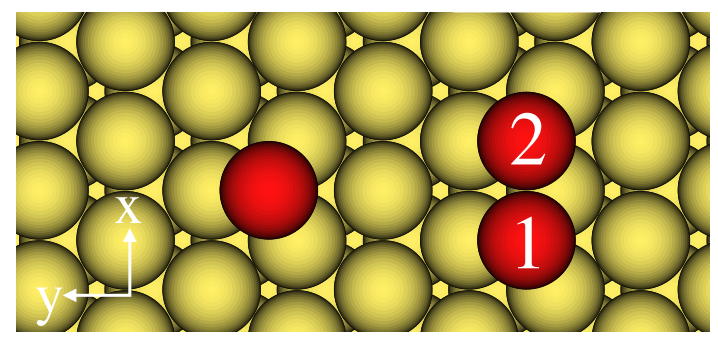

Fig. 1. Structures of the investigated systems: The cluster atoms occupy ideal lattice sites of the underlying Pt(111) substrate.

$$
\begin{aligned}
E(\theta, \phi)= & E_{0}+K_{2,1} \cos 2 \theta \\
& +K_{2,2}(1-\cos 2 \theta) \cos 2 \phi+K_{2,3} \sin 2 \theta \sin \phi
\end{aligned}
$$

Using a corresponding expression for the torque it is straight forward to deal with the integral occurring in Eq. (2). The evaluation of the anisotropy constants $K_{n, m}$ occurring in this equation can then be determined in a rather easy way by determining the torque for certain orentations $\hat{n}$ i.e. at angles $(\theta, \phi)$ of the magnetic moments (see below).

\section{Results and Discussion}

The structure of the investigated Co and Fe monomers and dimers are shown together with the underlying $\mathrm{Pt}(111)$ substrate in Fig. 11 As the ad- or cluster atoms, respectively occupy regular lattice sites correspondingly to the substrate the resulting cluster/substrate system has $\mathrm{C}_{3 \mathrm{v}^{-}}$ and $\mathrm{C}_{2 \mathrm{v}}$-symmetry, respectively. Comparing the resulting spin and orbital magnetic moments of $\mathrm{Co}$ and $\mathrm{Fe}$ in Tab. 1 one notices that the spin magnetic moments for $\mathrm{Fe}$ are in general about 1.5 times larger than for Co. For both transition metals the dimer formation has only a minor impact on their spin magnetic moments when compared to the single adatoms. The orbital moments, however, show a more interesting behaviour. Here we find already a substatial quenching when going from single adatoms to the corresponding pure i.e. unmixed dimers. This effect seems to be much more pronounced in the case of $\mathrm{Fe}$ where the orbital magnetic moment of a $\mathrm{Fe}_{2}$ dimer atom reduces to about one third of the monomer value compared to only three quarters in the case of Co. The corresponding values for the mixed dimer differ only slightly from those of $\mathrm{Co}_{2}$ and $\mathrm{Fe}_{2}$, respectively. The different behaviour of the orbital moment for $\mathrm{Co}$ and $\mathrm{Fe}$ is also reflected in their anisotropies (see below). A further increase in cluster size leads usually (depending also on the cluster shape) to a rapid and monotonous decay of spin and orbital magnetic moments approaching rapidly the values of a monolayer.

Fig. 2 shows the dependence of the $\theta$-component of the magnetic torque $T_{\theta}(\theta, \phi)=-\frac{\partial E}{\partial \theta}$ on the polar angle $\phi$ for $\mathrm{Co}$ and $\mathrm{Fe}$ monomers at $\theta=\frac{\pi}{4}$. As $T_{\theta}(\theta, \phi)$ is found to be negative here as well as for the following we show 
Table 1. Spin and orbital moments per atom of the investigated systems with magnetisation along the z-axis, i.e. perpendicular to the surface. For the mixed cluster the value refers to the component underlined.

\begin{tabular}{lcccccc}
\hline & $\mathrm{Co}_{1}$ & $\mathrm{Fe}_{1}$ & $\mathrm{Co}_{2}$ & $\mathrm{Fe}_{2}$ & $\mathrm{FeCo}$ & $\underline{\mathrm{FeCo}}$ \\
\hline$\mu_{\text {spin }}\left(\mu_{\mathrm{B}}\right)$ & 2.27 & 3.49 & 2.15 & 3.33 & 2.12 & 3.38 \\
$\mu_{\text {orb }}\left(\mu_{\mathrm{B}}\right)$ & 0.60 & 0.77 & 0.44 & 0.24 & 0.39 & 0.26 \\
\hline
\end{tabular}
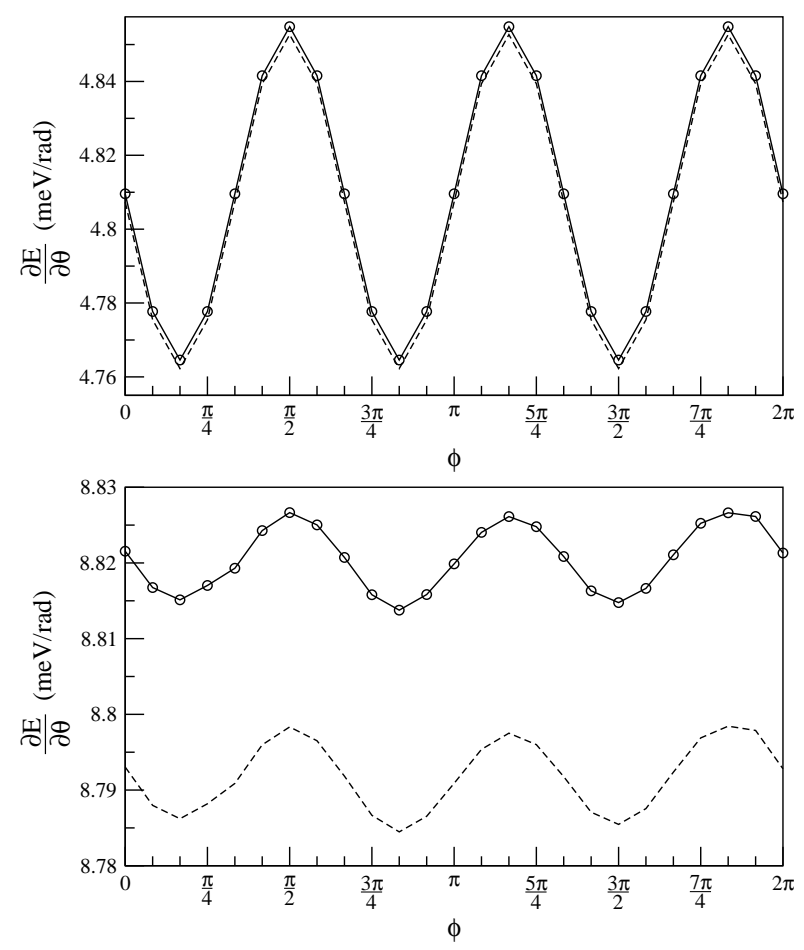

Fig. 2. Torque component $T_{\theta}(\theta, \phi)$ for $\mathrm{Co}_{1}$ (top) and $\mathrm{Fe}_{1}$ (bottom) at $\theta=\frac{\pi}{4}$ as a function of the polar angle $\phi$ (In fact $-T_{\theta}(\theta, \phi)=\frac{\partial E}{\partial \theta}$ is shown - see text). The dashed line shows the same results including the substrate effect.

always $\frac{\partial E}{\partial \theta}=-T_{\theta}(\theta, \phi)$. The positive sign of $\frac{\partial E}{\partial \theta}$ for all angles $\phi$ implies that the torque forces the magnetisation to the z-axis. This means that the system's easy axis points out of plane along $\hat{z}$. One can see that the threefold symmetry imposed by the underlying Pt substrate is directly reflected by the small oscillations of $T_{\theta}$ with the polar angle $\phi$.

This also demonstrates the high sensitivity of our implementation. The curves show no numerical noise even for an energy resolution below $0.1 \mathrm{meV}$. As the $\phi$ dependence of $T_{\theta}$ is so small when compared to its absolute value the adatoms almost behave like perfect uniaxial magnets. In this case the anisotropy constants $K$ can be extracted from the minima of $T_{\theta}(\theta, \phi)$. For the single adatoms this gives then $4.76 \mathrm{meV}$ for $\mathrm{Co}$ and $8.79 \mathrm{meV}$ for Fe. Fig. 2 also shows the influence of the induced anisotropy coming from the Pt substrate atoms. This induced MAE is about $-30 \mu \mathrm{eV}$ for Fe and even smaller in the case of Co. In fact it seems to be negligible for very small Co clusters composed
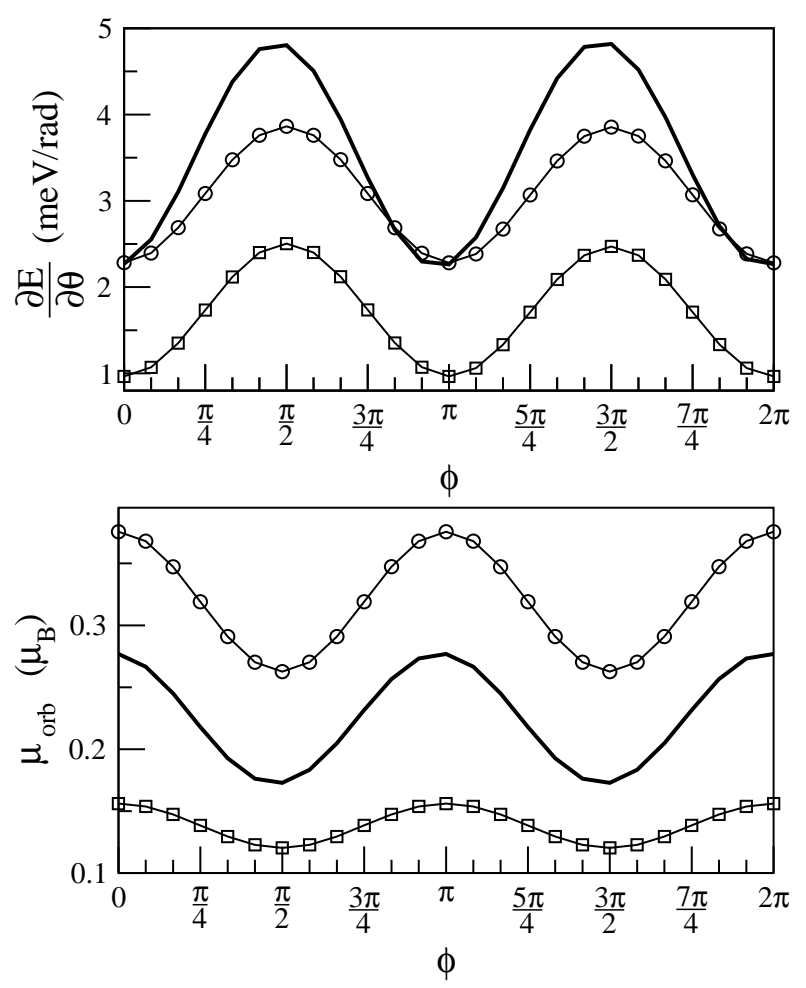

Fig. 3. Top panel: torque component $T_{\theta}(\theta, \phi)$ for $\mathrm{Co}_{2}$ (circles), $\mathrm{Fe}_{2}$ (squares) and the FeCo dimer (thick line) for $\theta=\frac{\pi}{4}$. Lower panel: $\phi$ dependence of the average orbital moments for all three dimers at $\theta=\frac{\pi}{2}$.

of only few atoms. For larger two-dimensional Co clusters, however, we found that this induced anisotropy becomes more important with increasing cluster size and can rise to the same order of magnitude as the contribution coming from the Co atoms themselves.

The results of the torque calculations for the three different investigated dimers are shown in the top panel of Fig. 3. In all cases $\frac{\partial E}{\partial \theta}$ is again positive, however, the absolute values are significantly reduced when compared to the monomers. The $\phi$-dependence of the $\theta$-component of the torque on the other hand is now much more pronounced due to the reduced symmetry of the cluster/substrate system when compared to the monomer (see Fig. 1). From Fig. 3 one can see that $E(\hat{n}, \hat{z})$ is smallest if the magnetic moments are oriented along the $\hat{x}$ direction, i. e. along the cluster dimer axis (see Fig. 1). The lower panel of Fig. 3 shows the corresponding $\phi$ dependence of the orbital moments for $\theta=\frac{\pi}{2}$. One can see that the oscillations in the orbital moments follow the oscillations of $T_{\theta}$ in an anticyclic manner. Here it should be pointed out that the largest values for the orbital moments are obtained when the magnetisation points along the $\mathrm{z}$-axis (see Table 11). This as well as the behaviour seen in Fig. 3] is in qualitative agreement with the Bruno and van der Laan anisotropy models 2122 that relate the MAE $E\left(\hat{n}, \hat{n}_{0}\right)$ to the corresponding anisotropy of the orbital moment.

For the van der Laan model one has 

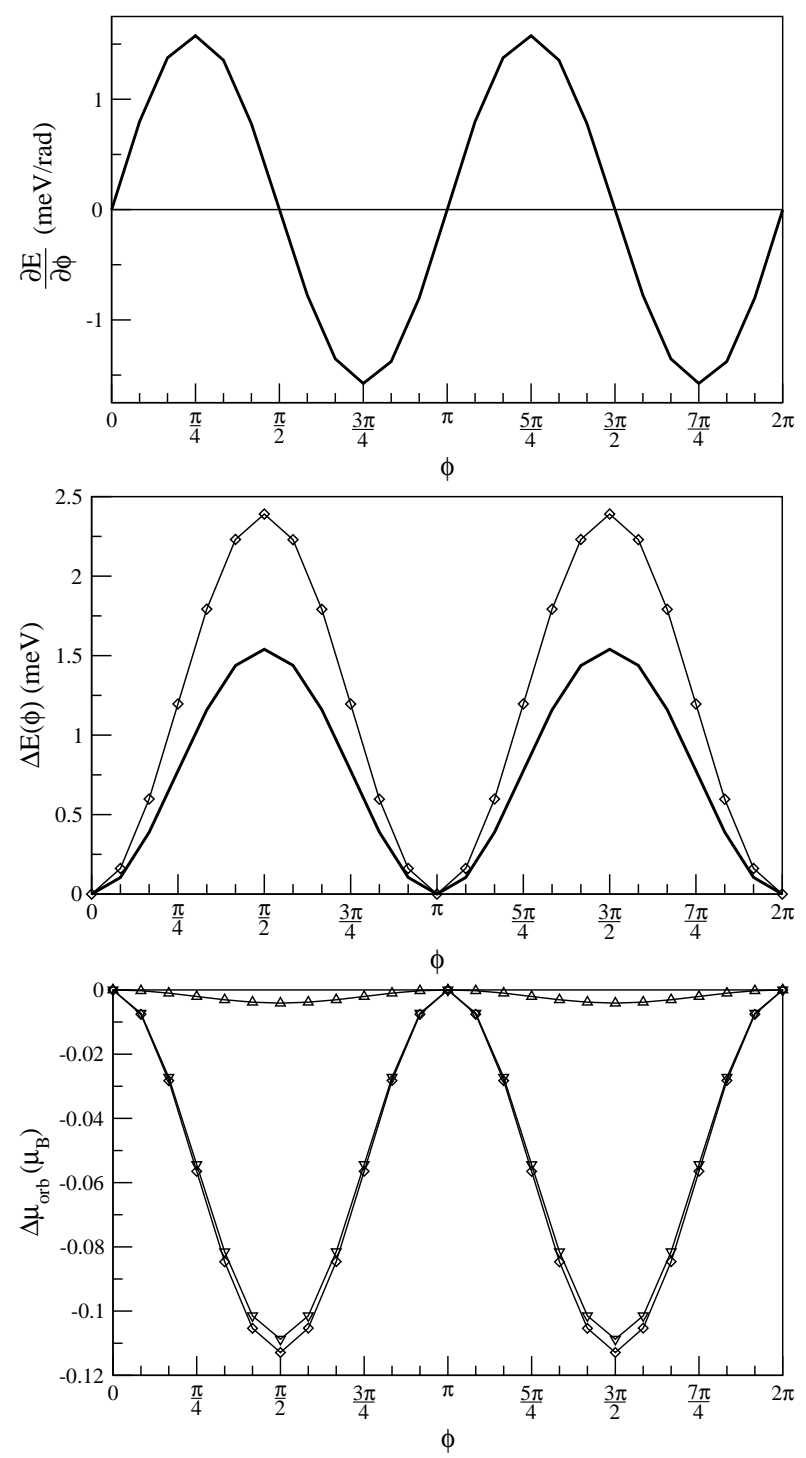

Fig. 4. Top panel: Torque component $T_{\phi}(\theta, \phi)$ of $\mathrm{Co}_{2}$ for $\theta=\frac{\pi}{2}$ as a fuction of $\phi$. Middle panel: MAE $\Delta E(\phi)$ for $\theta=\frac{\pi}{2}$ refered to $\hat{n}_{0} \hat{=}\left(\frac{\pi}{2}, \phi=0\right)$. The thick line gives calculated results while the thin line marked by diamonds gives results based on van der Laan's model. Lower Panel: spin resolved anisotropy $\Delta \mu^{m s}(\phi)$ for $\theta=\frac{\pi}{2}$ refered to $\hat{n}_{0} \hat{=}\left(\frac{\pi}{2}, \phi=0\right)$ for spin up and down (triangles up and down, resp.) and summed up (diamonds).

$$
\begin{aligned}
E\left(\hat{n}, \hat{n}_{0}\right)= & -\frac{\xi}{4 \mu_{B}}\left[\mu_{\text {orb }}^{\uparrow}(\hat{n})-\mu_{\text {orb }}^{\downarrow}(\hat{n})\right] \\
& +\frac{\xi}{4 \mu_{B}}\left[\mu_{\text {orb }}^{\uparrow}\left(\hat{n}_{0}\right)-\mu_{\text {orb }}^{\downarrow}\left(\hat{n}_{0}\right)\right]
\end{aligned}
$$

where $\xi$ is the appropriate spin-orbit coupling constant (here for the $3 \mathrm{~d}$ transition metal Fe or Co, respectively), and $\mu_{\mathrm{orb}}^{m_{s}}(\hat{n})$ is the spin projected orbital magnetic moment for an orientation of the magnetisation along $\hat{n}$. If one considers a strong ferromagnet one can ignore the majority spin contribution in Eq. (4) leading to the expectation

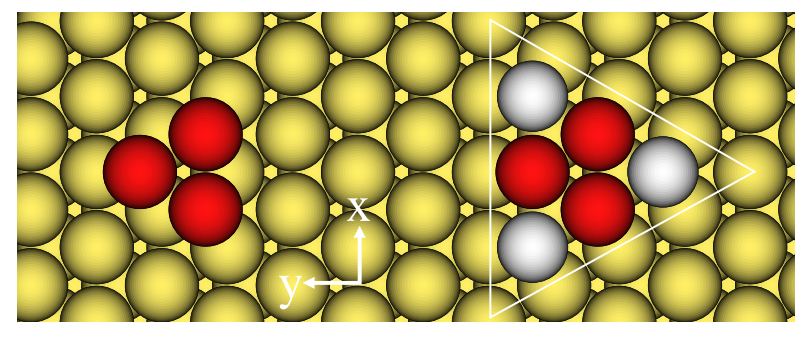

Fig. 5. Structure of the studied Fe trimer on $\operatorname{Pt}(111)$ without (left) and with (right) decoration by three Pt atoms.

that the orbital magnetic moment takes its maximum for the magnetisation oriented along the easy axis. The results shown in Figs. 2 and 3 are obviously in full agreement with this. As Fig. 3 shows for the three considered dimers, also the MAE deduced from the shown torque goes parallel with the orbital magnetic moment (lower panel) when $\phi$ is varied while $\theta$ is kept fixed.

The relation of the MAE and the anisotropy of the orbital magnetic moment has been studied in more detail by calculating the $\phi$-component of the torque for $\theta$ fixed to $\frac{\pi}{2}$, i.e. for the magnetisation forced to lie in the surface xy-plane. As one notes from the results given in Fig. 4, the variation of the torque is again quite pronounced and reflects the $\mathrm{C}_{2 \mathrm{v}}$-symmetry of the dimer/substrate system. Integrating the torque component with respect to $\phi$ (see Eq. (2)) one is led to the MAE $\Delta E\left(\left(\frac{\pi}{2}, \phi\right),\left(\frac{\pi}{2}, \phi\right)\right)$ shown in the middle panel of Fig. 4 , that again shows that the energy is at its minimum if the magnetisation lies along the dimer axis. The corresponding anisotropy $\Delta \mu(\phi)$ is given in the lower panel of Fig. 4 in a spin-polarized way. As one notes the majority contribution to $\Delta \mu_{\mathrm{orb}}(\phi)=$ $\mu_{\text {orb }}\left(\frac{\pi}{2}, \phi\right)-\mu_{\text {orb }}\left(\frac{\pi}{2}, 0\right)$ is very small and negligible. This is a consequence of the nearly complete filling of the majority spin d-band of the Co-atoms, i.e. their strong ferromagnetic behaviour. As a consequence, one has not to distinguish between Bruno's and van der Laan's models. Using the relation given in Eq. (4) together with the spin orbit coupling strength $\xi=85 \mathrm{meV}$ for $\mathrm{Co}[23$ one is lead to the estimate for the MAE $\Delta E(\phi)$ in the middle panel represented by the curve marked by diamonds. Obviously, the qualitative behaviour of the MAE is properly reproduced with a reasonable quantitative agreement.

Figs. 2 and 3 show that going from a monomer to a dimer the MAE is drastically reduced. This goes on with increasing cluster size, although there is some influence of the cluster shape 4. As one notes, the decrease of the MAE is much more pronounced for Fe than for Co. For the mixed FeCo cluster, however, the MAE is quite high. This indicates that by a suitable combination of atoms one may optimize the MAE while keeping the magnetisation high. In fact, there are already a number of experimental studies done along this line. Here, we show results for the impact on the properties of a Fe trimer on $\mathrm{Pt}(111)$ due to a decoration with $\mathrm{Pt}$ atoms.

Fig. 5 shows the corresponding structure of the investigated cluster/substrate systems. The resulting spin and orbital magnetic moments are given in Table 2, As one 
Table 2. Spin and orbital moments per atom of the $\mathrm{Fe}_{3}$ and $\mathrm{Fe}_{3} \mathrm{Pt}_{3}$ clusters on $\mathrm{Pt}(111)$ for the magnetisation along the $\mathrm{z}-$ axis, i.e. perpendicular to the surface. The values refer to the component underlined. The last two columns give results for the Pt-substrate atoms having one or two Fe atoms as nearest neighbours, resp., in case of $\mathrm{Fe}_{3} \mathrm{Pt}_{3}$.

\begin{tabular}{lccccc}
\hline & $\mathrm{Fe}_{3}$ & $\underline{\mathrm{Fe}}_{3} \mathrm{Pt}_{3}$ & $\mathrm{Fe}_{3} \underline{\mathrm{Pt}}_{3}$ & $\mathrm{Pt}(1)$ & $\mathrm{Pt}(2)$ \\
\hline$\mu_{\text {spin }}\left(\mu_{\mathrm{B}}\right)$ & 3.20 & 3.16 & 0.21 & 0.09 & 0.11 \\
$\mu_{\text {orb }}\left(\mu_{\mathrm{B}}\right)$ & 0.16 & 0.09 & 0.08 & 0.02 & 0.02 \\
\hline
\end{tabular}

notes, the moments of the $\mathrm{Fe}$ atoms are again quite high as for the mono- and dimer. For the spin moment one finds only a minor influence due to the decorating Pt atoms. These have also quite an appreciable induced magnetic moment. The resulting torque $T_{\theta}(\theta, \phi)$ for $\theta=\frac{\pi}{4}$ as a function of the angle $\phi$ is shown in Fig. 6 (top panel) for the $\mathrm{Fe}_{2-}, \mathrm{Fe}_{3^{-}}$and $\mathrm{Fe}_{3} \mathrm{Pt}_{3}$ clusters. As is demonstrated once more the torque $T_{\theta}$ and with this also the MAE is strongly reduced going from the dimer to the trimer. Adding the decoration, however, the high MAE of the dimer is recovered. As the inner $\mathrm{Fe}_{3}$ cluster of the $\mathrm{Fe}_{3} \mathrm{Pt}_{3}$ cluster is now surrounded by neighbouring atoms the variation of the MAE with $\phi$ is strongly reduced when compared with the dimer.

As one would expect, the MAE of the decorated trimer $\mathrm{Fe}_{3} \mathrm{Pt}_{3}$ is dominated by its Fe contribution. This can be seen in the middle panel of Fig. 6 where apart from the total MAE per Fe-atom the averaged contribution of a Featom is shown. Nevertheless, there is an appreciable contribution coming from the decorating $\mathrm{Pt}$ atoms as well. This has to be ascribed on the one-hand side to their appreciable induced magnetic moment (see Tab. 2) and on the other side to their large spin-orbit coupling $\left(\xi_{\mathrm{Pt}}=710\right.$ $\mathrm{meV}$ to be compared with $\xi_{\mathrm{Fe}}=65 \mathrm{meV}$ ). Apart from the contribution of the decorating $\mathrm{Pt}$ atoms there is a noteworthy contribution from the neighbouring Pt-substrate atoms as well. As can be seen in the lower panel of Fig. [6 this amounts to about $5 \%$ of the total torque or MAE, respectively.

The results for the undecorated $\mathrm{Fe}_{n}$ clusters $(\mathrm{n}=1,2,3)$ shown in the lower panel of Fig. 2 and the top panel of Fig. 6 allows us to make contact to corresponding experimental investigations 24. The sample preparation technique used within these investigations led to an ensemble of $\mathrm{Fe}_{n}$ clusters that was dominated by clusters of size $\mathrm{n}=1$ 3 with their statistical weight determined to be $\mathrm{w}_{n}=0.53$, 0.54 and $0.33 \%$ (for technical details see Ref. [7]). Fig. 7 shows the magnetisation curves $M(B)$ measured for this cluster ensemble at $\mathrm{T}=6 \mathrm{~K}$ for an orientation of the external magnetic field $\mathbf{B}$ along the easy axis $(\hat{z})$ and at an angle $\theta \hat{=} 65^{\circ}$ with respect to this axis. With the magnetic moments and the anisotropy parameters for the $\mathrm{Fe}_{n}$ clusters available the magnetisation curves $M(B)$ can be simulated by means of the so-called Langevin formula [4. This way the thermal average of the z-component $m_{n z}(B, T)$ of the moment $m_{n}$ of an $\mathrm{Fe}_{n}$ cluster can be expressed by:
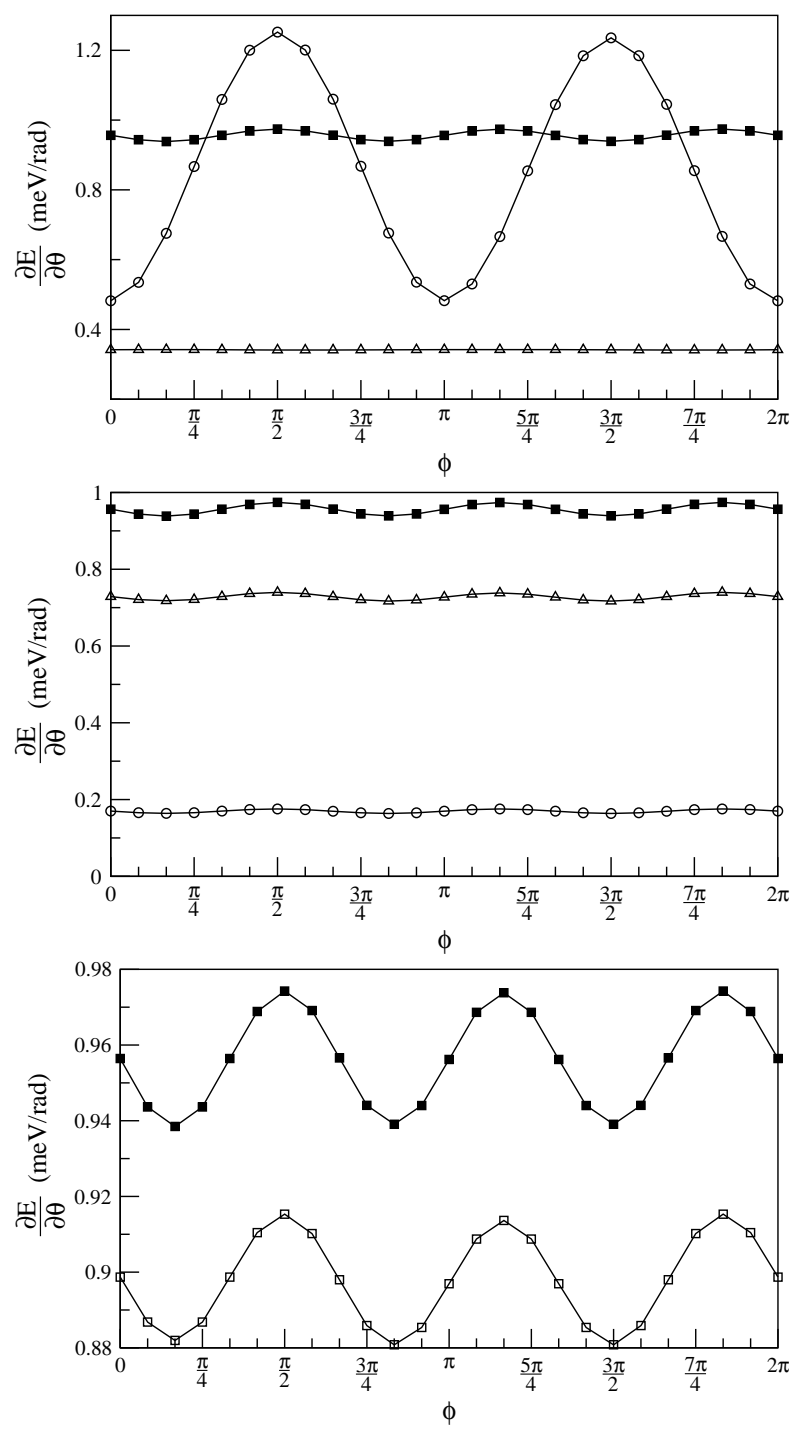

Fig. 6. Top panel: torque component $T_{\theta}(\theta, \phi)$ for $\mathrm{Fe}_{2}$ (circles), $\mathrm{Fe}_{3}$ (triangles) and $\mathrm{Pt}_{3} \mathrm{Fe}_{3}$ (squares) for $\theta=\frac{\pi}{4}$ as a function of the polar angle $\phi$. Middle Panel: torque component $T_{\theta}(\theta, \phi)$ per Fe atom for $\mathrm{Pt}_{3} \mathrm{Fe}_{3}$ at $\theta=\frac{\pi}{4}$ as a function of $\phi$ : total (full squares) including the substrate contribution, contribution of a Fe atom (triangle) and a cluster $\mathrm{Pt}$ atom (circles). Lower Panel: total torque component $T_{\theta}(\theta, \phi)$ per Fe atom for $\mathrm{Pt}_{3} \mathrm{Fe}_{3}$ at $\theta=\frac{\pi}{4}$ as a function of the polar angle $\phi$ with (full squares) and without (open squares) the substrate contribution.

$$
m_{n z}(B, T)=\frac{\int_{0}^{\pi} \sin \theta d \theta e^{-E(B, T, \theta) / k T} m_{n} \cos \theta}{\int_{0}^{\pi} \sin \theta d \theta e^{-E(B, T, \theta) / k T}} .
$$

For the simulation the energy $E(B, T, \theta)$ was assumed to consist of its Zeeman and anisotropy contributions

$$
E(B, T, \theta)=B m_{n} \cos \theta+K_{1}^{n} \sin ^{2} \theta
$$

where for the later one an uniaxial behaviour has been assumed. The corresponding anisotropy constant $K_{1}^{n}$ has 


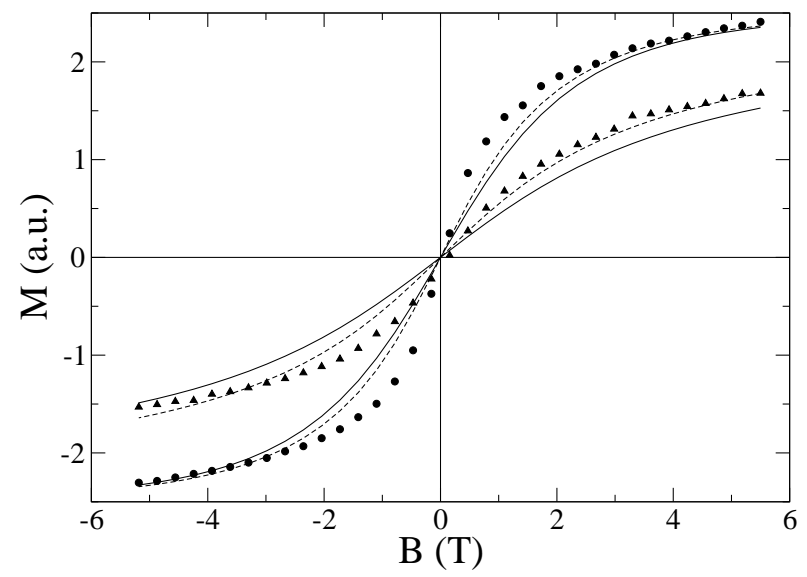

Fig. 7. Experimental magnetisation curves $M(B)$ (dots) of an ensemble if $\mathrm{Fe}_{n}$ clusters on $\mathrm{Pt}(111)$ measured at $\mathrm{T}=6 \mathrm{~K}$ for an orientation of the magnetic field $M(B)$ along the easy axis $\hat{z}\left(\theta=0^{\circ}\right)$ and rotated by $\theta=65^{\circ}$ with respect to that. The full lines give corresponding theoretical results obtained on the basis of the calculated properties of $\mathrm{Fe}_{n}$ clusters and the Langevin formula given in Eq. (5). The dashed line is obtained by including $\mathrm{Fe}_{4}$ clusters in the simulation.

been deduced from the results for the MAE shown above. Adding the magnetisation curves for the $\mathrm{Fe}_{n}$ clusters weighted by their statistical weight $\mathrm{w}_{n}$ one is lead to the full lines shown in Fig. 7. The additional dashed lines stem from a second simulation done including tetramers. This indicates that a certain amount of larger Fe clusters are formed during the preparation process as expected by statistics.

The good agreement of the simulated curves with experiment obviously demonstrates that the complex magnetic properties of transition metal clusters can indeed be understood and described without adjustable parameters on the basis of the approach used here. It also shows that inclusion of relaxation effects when calculating the cluster properties should only slightly modify the numerical results. Nevertheless, corresponding numerical works are in progress to determine the influence of lattice relaxations.

\section{Conclusion}

We have presented results for the magnetic anisotropy of various $\mathrm{Co} / \mathrm{Pt}(111)$ and $\mathrm{Fe} / \mathrm{Pt}(111)$ cluster/substrate systems calculated by the fully relativistic KKR-approach. It was demonstrated that calculating the magnetic torque instead of the magnetic anisotropy energy directly, is numerical very robust and reliable. This is in particular reflected by the accuracy achieved for the dependency of the torque on the polar angle $\phi$ as well as the results for the substrate contribution.

As in previous work it was found that increasing the cluster size in general a rapid decrease of the MAE occurs. However, it could be shown that by suitable formation of compound clusters this drop can be compensated. In fact, the combination of an element with large magnetic moments with one having large spin-orbit coupling seems to be a promising approach.

Finally, it could be demonstrated that using the calculated cluster properties the results of experimental magnetisation curves could be reproduced in a very satisfying way confirming the adequateness of our approach as well as the interpretation of the experimental findings.

\section{Acknowledgement}

We acknowledge support by the Deutsche Forschungsgemeinschaft within the Schwerpunktprogramm 1153 Cluster in Kontakt mit Oberflächen: Elektronenstruktur und Magnetismus.

\section{References}

1. J. Bansmann, S.H. Baker, C. Binns, J.A. Blackman, J.P. Bucher, J. Dorantes-Davila, V. Dupuis, L. Favre, D. Kechrakos, A. Kleibert et al., Surface Science Reports 56, 189 (2005)

2. G. Nicolas, J. Dorantes-Davila, G.M. Pastor, Phys. Rev. B 74, 014415 (2006)

3. F. Meier, K. von Bergmann, P. Ferriani, J. Wiebe, M. Bode, K. Hashimoto, S. Heinze, R. Wiesendanger, Phys. Rev. B 74, 195411 (2006)

4. P. Gambardella, S. Rusponi, M. Veronese, S.S. Dhesi, C. Grazioli, A. Dallmeyer, I. Cabria, R. Zeller, P.H. Dederichs, K. Kern et al., Science 300, 1130 (2003)

5. S. Rusponi, T. Cren, N. Weiss, M. Epple, B. Buluschek, L. Claude, H. Brune, Nature Materials 2, 546 (2003)

6. C. Petit, S. Rusponi, H. Brune, J. Appl. Phys. 95, 4251 (2004)

7. P. Gambardella, A. Dallmeyer, K. Maiti, M.C. Malagoli, W. Eberhardt, K. Kern, C. Carbone, Nature 416, 301 (2002)

8. P. Gambardella, S. Rusponi, T. Cren, N. Weiss, H. Brune, C. R. Physique 6, 75 (2005)

9. H. Brune, e-J. Surf. Sci. Nanotech. 4, 478 (2006)

10. P. Gambardella, A. Dallmeyer, K. Maiti, M.C. Malagoli, S. Rusponi, P. Ohresser, W. Eberhardt, C. Carbone, K. Kern, Physical Review Letters 93, 077203 (2004)

11. O. Šipr, S. Bornemann, J. Minár, S. Polesya, V. Popescu, A. Simunek, H. Ebert, J. Phys.: Condensed Matter p. in press (2006)

12. S. Bornemann, J. Minár, S. Polesya, S. Mankovsky, H. Ebert, O. Šipr, Phase Transitions 78, 701 (2005)

13. J. Minár, S. Bornemann, O. Šipr, S. Polesya, H. Ebert, Appl. Physics A 82, 139 (2006)

14. B. Lazarovits, L. Szunyogh, P. Weinberger, Phys. Rev. B 67, 24415 (2003)

15. Y. Xie, J.A. Blackman, Phys. Rev. B 74, 054401 (2006)

16. S.H. Vosko, L. Wilk, M. Nusair, Can. J. Phys. 58, 1200 (1980)

17. H. Ebert, Fully relativistic band structure calculations for magnetic solids - Formalism and Application, in Electronic Structure and Physical Properties of Solids, edited by H. Dreyssé (Springer, Berlin, 2000), Vol. 535 of Lecture Notes in Physics, p. 191 
18. H. Ebert and R. Zeller, The SPR-TB-KKR package, http://olymp.cup.uni-muenchen.de/ak/ebert/SPR-TBKKR (2005)

19. J.B. Staunton, L. Szunyogh, A. Buruzs, B.L. Gyorffy, S. Ostanin, L. Udvardi, Phys. Rev. B 74, 144411 (2006)

20. B. Lazarovits, B. Ujfalussy, L. Szunyogh, G.M. Stocks, P. Weinberger, J. Phys.: Condensed Matter 16, S5833 (2004)

21. P. Bruno, Phys. Rev. B 39, 865 (1989)

22. G. van der Laan, J. Phys.: Condensed Matter 10, 3239 (1998)

23. V. Popescu, H. Ebert, B. Nonas, P.H. Dederichs, Phys. Rev. B 64, 184407 (2001)

24. D. Repetto, T.Y. Lee, S. Rusponi, J. Honolka, K. Kuhnke, V. Sessi, U. Starke, H. Brune, P. Gambardella, C. Carbone et al., Phys. Rev. B 74, 054408 (2006) 